\title{
A combinatorial proof of Klyachko's Theorem on Lie representations
}

\author{
L. G. Kovács · Ralph Stöhr
}

Received: March 28, 2005 / Revised: July 12, 2005 / Accepted: September 2, 2005

(C) Springer Science + Business Media, Inc. 2006

\begin{abstract}
Let $L$ be a free Lie algebra of finite rank $r$ over an arbitrary field $K$ of characteristic 0 , and let $L_{n}$ denote the homogeneous component of degree $n$ in $L$. Viewed as a module for the general linear group $G L(r, K), L_{n}$ is known to be semisimple with the isomorphism types of the simple summands indexed by partitions of $n$ with at most $r$ parts. Klyachko proved in 1974 that, for $n>6$, almost all such partitions are needed here, the exceptions being the partition with just one part, and the partition in which all parts are equal to 1 . This paper presents a combinatorial proof based on the Littlewood-Richardson rule. This proof also yields that if the composition multiplicity of a simple summand in $L_{n}$ is greater than 1 , then it is at least $\frac{n}{6}-1$.
\end{abstract}

Keywords Free Lie algebra · General linear group · Littlewood-Richardson rule

Let $V$ be a finite dimensional vector space over an arbitrary field of characteristic 0 , and let $T$ be the the tensor algebra of $V$, so $T=\bigoplus_{n \geq 0} T_{n}$ with $T_{n}=V^{\otimes n}$. Recall that the tensor powers $T_{n}$ are semisimple $G L(V)$-modules, and the isomorphism types of the simple submodules of $T_{n}$ correspond to the partitions of $n$ into not more than $\operatorname{dim} V$ parts. The tensor product of any two such irreducibles is then also a direct sum of such irreducibles, and the relevant multiplicities are given by the Littlewood-Richardson rule.

Consider $T$ a Lie algebra with respect to the Lie product $[x, y]=x \otimes y-y \otimes x$, and denote by $L$ the Lie subalgebra generated by $V\left(=T_{1}\right)$. Then $L$ is freely generated by any basis of $V, L=\bigoplus_{n \geq 1} L_{n}$ with $L_{n}=L \cap T_{n}$, and $L_{n}$ is a submodule of $T_{n}$. In 1942, Thrall [12] asked for the composition multiplicities of these modules, and determined them for $n \leq 10$ (for $n=10$, a correction was given in [1]). By 1949, Wever [14] gave a formula for these multiplicities in terms of characters of symmetric groups; nowadays, the highly

\section{G. Kovács}

Mathematics, Australian National University, Canberra ACT 0200, Australia

e-mail: kovacs@maths.anu.edu.au

R. Stöhr $(\bowtie)$

School of Mathematics, University of Manchester, PO Box 88, Manchester M60 1QD, United Kingdom ralph.stohr@manchester.ac.uk 
illuminating Kraśkiewicz-Weyman Theorem [5] (see also Chapter 8 in [8]) may be invoked for an answer in terms of counting tableaux of certain kinds. Nevertheless, the work started by Thrall still continues today, with the scope of the problem having increased greatly; for further references and a recent overview, see [9].

A 1974 paper [4] of Alexander Klyachko provided a large impetus, and included the following remarkable result.

Theorem 1. [Klyachko [4]] Let $n \geq 3$ and let $v$ be a partition of $n$. There is a simple submodule in $L_{n}$ with isomorphism type corresponding to $v$ if and only if $v$ has no more than $\operatorname{dim} V$ parts and $v$ is not one of $\left(2^{2}\right),\left(2^{3}\right),(n),\left(1^{n}\right)$.

The notation used here is best explained by an example: $\left(2,1^{2}\right)$ denotes the 3 -part partition $2+1+1$ of 4 . If $v=\left(2,1^{2}\right)$, we shall write the corresponding module simply as $[v]$ or $\left[2,1^{2}\right]$. The reader is expected to interpret everything that follows in the light of the convention that the $G L(V)$-module corresponding to a partition with more than $\operatorname{dim} V$ parts is 0 , so then there is no such simple module.

The multiplicity formulas mentioned above are very useful when one wants to deal with one multiplicity at a time, but do not seem to help in proving global results like Klyachko's Theorem (or some others mentioned at the end of this note). As Schocker [10, p. 286] notes, "it seems to be rather difficult to give a combinatorial proof [...] by some analysis [...] and the Kraśkiewicz-Weyman Theorem only." There have been other proofs, perhaps the latest by Schocker [9, 10], built on the important developments which started with [4]. The aim of this note is to present a proof which does not do so, but relies only on the Littlewood-Richardson rule and on simple properties of free Lie algebras, and which has some further consequences. It is based on the following observation.

Lemma 1. Let $n=k+l$ with $k>l>k / 2$. The subspace $\left[L_{k}, L_{l}\right]$ of $L_{n}$ spanned by the $[u, w]$ with $u \in L_{k}, w \in L_{l}$ is a submodule isomorphic to the tensor product $L_{k} \otimes L_{l}$.

Proof: We shall argue in terms of a Hall basis $\mathcal{H}$ of $L$, but first we need to set the relevant conventions, for standard sources vary in their choices. We follow Marshall Hall's original paper [2]. There $\mathcal{H}$ consists of homogeneous elements of $L$ (so $\mathcal{H} \cap L_{m}$ is always a basis of $\left.L_{m}\right)$ and is fully ordered by a relation $\leq$ which extends the partial order given by degrees. Every element of $\mathcal{H}$ of degree greater than 1 can be written uniquely in the form $[u, w]$ where $u, w \in \mathcal{H}$ and $u>w$. Finally, if $u, w \in \mathcal{H}$ and $u>w$, then $[u, w] \in \mathcal{H}$ if and only if either the degree of $u$ is 1 or $u=\left[u^{\prime}, u^{\prime \prime}\right]$ with $u^{\prime}, u^{\prime \prime} \in \mathcal{H}$ and $u^{\prime}>u^{\prime \prime} \leq w$.

Let us turn to the proof of the lemma itself. Since $G L(V)$ acts on $L$ by Lie algebra automorphisms, the linear extension $L_{k} \otimes L_{l} \rightarrow L_{n}$ of $u \otimes w \mapsto[u, w]$ is in fact a module homomorphism. The set $\left\{u \otimes w \mid u \in \mathcal{H} \cap L_{k}, w \in \mathcal{H} \cap L_{l}\right\}$ is a basis for $L_{k} \otimes L_{l}$; call it $\mathcal{B}$, say. We claim that the image $[u, w]$ of an element $u \otimes w$ of $\mathcal{B}$ is always in $\mathcal{H}$. The first part of this claim is that $u>w$ : this holds because $k>l$. The second part is that if $u=\left[u^{\prime}, u^{\prime \prime}\right]$ with $u^{\prime}, u^{\prime \prime} \in \mathcal{H}$, then $u^{\prime \prime} \leq w$. In fact, $u^{\prime \prime}<w$, because $u \in \mathcal{H}$ implies $u^{\prime}>u^{\prime \prime}$ and so the degree of $u^{\prime \prime}$ is at most $k / 2$ and hence strictly smaller than the degree $l$ of $w$. It follows that our module homomorphism maps the basis $\mathcal{B}$ of its domain into a basis, $\mathcal{H} \cap L_{n}$, of its codomain. Moreover, its restriction to $\mathcal{B}$ is one-to-one, because the expression of an element of $\mathcal{H}$ in the form $[u, w]$ with $u, w \in \mathcal{H}$ and $u>w$ is unique. Consequently, the image of this homomorphism is isomorphic to its domain. 
The Littlewood-Richardson rule makes it possible to exploit this in an inductive argument. To start that off, we need to recall some of the information tabulated by Thrall [12] for the $L_{n}$ with small $n$ :

$$
\begin{aligned}
& L_{1} \cong[1], \quad L_{2} \cong\left[1^{2}\right], \quad L_{3} \cong[2,1], \quad L_{4} \cong[3,1] \oplus\left[2,1^{2}\right], \\
& L_{5} \cong[4,1] \oplus[3,2] \oplus\left[3,1^{2}\right] \oplus\left[2^{2}, 1\right] \oplus\left[2,1^{3}\right], \\
& L_{6} \cong[5,1] \oplus[4,2] \oplus\left[4,1^{2}\right]^{\oplus 2} \oplus\left[3^{2}\right] \oplus[3,2,1]^{\oplus 3} \oplus\left[3,1^{2}\right] \oplus\left[2^{2}, 1^{2}\right]^{\oplus 2} \oplus\left[2,1^{4}\right] .
\end{aligned}
$$

Next we have to deal with the extreme cases. These will need only very special cases of the Littlewood-Richardson rule. One, that the simple modules which occur in $[\kappa] \otimes[\lambda]$ all correspond to partitions which are extensions of $\kappa$ (and of $\lambda$, of course). Two, that if $\kappa$ is a partition of $k$, then $[\kappa] \otimes[1]$ is the direct sum of one copy each of the [v] as $v$ ranges over the partitions of $k+1$ which are extensions of $\kappa$.

Our notation for Lie products follows the left-normed convention: $[u, v, w]$ stands for [[u,v],w], etc. Since $L_{n}$ is spanned by the $[u, v]$ with $u \in L_{n-1}, v \in V$, the linear extension of $u \otimes v \mapsto[u, v]$ is a $G L(V)$-homomorphism of $L_{n-1} \otimes V$ onto $L_{n}$. We shall find this useful in proving the next result.

Lemma 2. For $n \geq 3$, neither of the simple modules $[n]$ and $\left[1^{n}\right]$ can occur in $L_{n}$, and neither $[n-1,1]$ nor $\left[2,1^{n-2}\right]$ can occur with multiplicity greater than 1 .

Proof: The list (1) provides the inital step for an induction on $n$, so we proceed to the inductive step. The partition $(n+1)$ is not an extension of any partition of $n$ except $(n)$; by the inductive hypothesis, $[n]$ does not occur in $L_{n}$, so $[n+1]$ cannot occur in $L_{n} \otimes V$; as $L_{n+1}$ is a homomorphic image of this tensor product, $[n+1]$ cannot occur in $L_{n+1}$ either. The partition $(n, 1)$ is not an extension of any partition of $n$ other than $(n)$ and $(n-1,1)$; by the inductive hypothesis, $[n]$ does not occur in $L_{n}$ and $[n-1,1]$ occurs at most once, so $[n, 1]$ cannot occur in $L_{n} \otimes V$ with multiplicity greater than 1 ; as $L_{n+1}$ is a homomorphic image of this tensor product, the multiplicity of $[n, 1]$ in $L_{n+1}$ cannot be larger either. The other cases are similar.

Let $\circ$ and $\wedge$ denote symmetric and exterior products, respectively, and recall that $[n]$ is the symmetric power $V^{\circ n}$ while $\left[1^{n}\right]$ is the exterior power $V^{\wedge n}$.

Lemma 3. For $n \geq 3$, the simple module $[n-1,1]$ occurs in $L_{n}$ provided $\operatorname{dim} V \geq 2$, and $\left[2,1^{n-2}\right]$ also occurs if $\operatorname{dim} V \geq n-1$.

Proof: There is a module homomorphism $L_{n} \rightarrow V \otimes V^{\circ(n-1)}$ such that

$$
\left[v_{1}, v_{2}, v_{3}, \ldots, v_{n}\right] \mapsto v_{1} \otimes\left(v_{2} \circ v_{3} \circ \cdots \circ v_{n}\right)-v_{2} \otimes\left(v_{1} \circ v_{3} \circ \cdots \circ v_{n}\right)
$$

(see [3, Theorem 3.1]), and this is clearly not zero when $\operatorname{dim} V \geq 2$. Since

$$
V \otimes V^{\circ(n-1)} \cong[1] \otimes[n-1] \cong[n] \oplus[n-1,1]
$$

and we have seen that $[n]$ does not occur in $L_{n}$, it follows that $[n-1,1]$ must occur in $L_{n}$. 
It is also well known (see Levin [6] or Vaughan-Lee [13]) that

$$
v_{1} \otimes\left(v_{2} \wedge \cdots \wedge v_{n}\right) \mapsto \sum_{\sigma} \operatorname{sgn}(\sigma)\left[v_{1}, v_{\sigma(2)}, \ldots, v_{\sigma(n)}\right]
$$

where $\sigma$ runs over all permutations of $\{2, \ldots, n\}$, extends to a nonzero homomorphism $V \otimes V^{\wedge(n-1)} \rightarrow L_{n}$ whenever $\operatorname{dim} V \geq n-1$. Since

$$
V \otimes V^{\wedge(n-1)} \cong[1] \otimes\left[1^{n-1}\right] \cong\left[1^{n}\right] \oplus\left[2,1^{n-2}\right]
$$

and we have seen that $\left[1^{n}\right]$ does not occur in $L_{n}$, we conclude that $\left[2,1^{n-2}\right]$ must occur in it.

Our last lemma concerns only $G L(V)$-modules, not Lie algebras, and may be of some interest in itself. It does need the full generality of the Littlewood-Richardson rule, though not its full force: instead of counting precise multiplicities, it is sufficient to know that the relevant multiplicities are positive. For a complete statement and the terminology not explained here, see Macdonald's book [7, pp. 4-5, 68].

When we call a diagram or a skew-diagram a rectangle, we use the word in its everyday sense. A partition will be called rectangular if its diagram is a rectangle, that is, if it is of the form $\left(r^{s}\right)$. Let $U_{n}$ denote the direct sum of the [ $\left.v\right]$ as $v$ ranges through the non-rectangular partitions of $n$. We shall use that if Klyachko's Theorem holds for a particular value of $n$, then for this value $L_{n}$ has a submodule isomorphic to $U_{n}$.

Lemma 4. Let $n=k+l$ where $k, l \geq 3$. If $v$ is a partition of $n$ other than $(n),(n-1,1)$, $\left(2,1^{n-2}\right)$, $\left(1^{n}\right)$, then $[v]$ does occur in $U_{k} \otimes U_{l}$.

Proof: First we show that at least one of $k$ and $l$ has a partition $\kappa$ such that $(*) \kappa$ is not rectangular, $\kappa \subset v$, and the skew diagram $v-\kappa$ is not a rectangle. If neither $k$ nor $l$ has a rectangular partition contained in $v$, then any partition $\kappa$ of $k$ contained in $v$ will do: indeed, if $v-\kappa$ were an $r \times s$ rectangle, then $\left(r^{s}\right)$ would be a rectangular partition of $l$ contained in $v$. Otherwise one of $k$ and $l$, say $k$, has a rectangular partition, say $\left(p^{q}\right)$, contained in $v$. If $q=1$, take $\kappa=(p-1,1)$ : the conditions on $v$ garantee that $\kappa \subset v$, and it is also easy to see that $v-\kappa$ is not a rectangle. Indeed, $v-\kappa$ contains the $1, p$ box and at least one of the 2, 2 or 3,1 boxes, but not the 2,1 box, so $v-\kappa$ is not convex and therefore it cannot be a rectangle. Similarly, if $p=1$, then we can take $\kappa=\left(2,1^{q-2}\right)$. Now suppose that $p, q \geq 2$. Then $v$ contains either the $1, p+1$ box or the $q+1,1$ box. In the former case $\kappa=\left(p+1, p^{q-2}, p-1\right)$ and in the latter case $\kappa=\left(p^{q-1}, p-1,1\right)$ will do.

Since the lemma is symmetric in $k$ and $l$, we may assume that $\kappa$ is a partition of $k$ satisfying (*). We claim that then there exists a non-rectangular partition $\lambda$ of $l=n-k$ such that $[\nu]$ occurs in $[\kappa] \otimes[\lambda]$. To see this, consider the tableau $T$ obtained by putting consecutive numbers $1,2, \ldots$ down each column of the skew diagram $v-\kappa$. It is easily seen that in this way we get a tableau for which $w(\mathrm{~T})$ is a lattice permutation. Let the weight of $\mathrm{T}$ be $\lambda$. Then the Littlewood-Richardson rule implies that $[v]$ occurs in $[\kappa] \otimes[\lambda]$. If $\lambda$ is not rectangular, we are done, so it remains to deal with the case where $\lambda$ is rectangular. This can only happen if $v-\kappa$ consists of columns of equal length, and there must be at least two columns since $v-\kappa$ is not a rectangle. Moreover, for the same reason we can find at least one column whose last box is strictly lower than the last box of the rightmost column. Take the last of those columns, and modify $T$ and $\lambda$ by adding 1 to the entry in its last box. 
This ensures that $\lambda$ is not rectangular, while the word $w(\mathrm{~T})$ is still a lattice permutation. Again, the Littlewood-Richardson rule implies that $[\nu]$ occurs in $[\kappa] \otimes[\lambda]$, and hence in $U_{k} \otimes U_{l}$

Proof of Klyachko's Theorem: In view of the list (1), the theorem is valid for $n \leq 6$. For a proof by induction on $n$, we may therefore assume that $n \geq 7$. Then one can write $n$ as a $\operatorname{sum} n=k+l$ with $k>l>k / 2$ and $k, l \geq 3$. By Lemma $1, L_{n}$ has a submodule isomorphic to the tensor product $L_{k} \otimes L_{l}$; by the inductive hypothesis, the theorem holds for $L_{k}$ and $L_{l}$, so $L_{k} \otimes L_{l}$ contains $U_{k} \otimes U_{l}$; therefore, by Lemma 4, every [v] occurs in $L_{n}$ except perhaps $[n],[n-1,1],\left[2,1^{n-2}\right]$ and $\left[1^{n}\right]$. Finally, $[n-1,1]$ and $\left[2,1^{n-2}\right]$ do occur by Lemma 3 , while $[n]$ and $\left[1^{n}\right]$ do not, by Lemma 2 . This completes the inductive step.

Remark. We have proved more than Klyachko's Theorem, namely (see Lemmas 2 and 3) that the multiplicities of $[n-1,1]$ and $\left[2,1^{n-2}\right]$ in $L_{n}$ are 0 or 1 , depending only on $\operatorname{dim} V$. This was proved by Zhuravlev $[15, \S 4]$ and by Schocker $[9,10]$. In $[9,10]$, it was also shown that no other multiplicity is 1 when $n>8$. Instead of pursuing that here, we note that it is easy to modify the proof of Lemma 1 to show that in $L_{n}$

$$
\sum_{n / 2<k<2 n / 3}\left[L_{k}, L_{n-k}\right] \cong \bigoplus_{n / 2<k<2 n / 3}\left(L_{k} \otimes L_{n-k}\right) .
$$

(Indeed, if $\mathcal{H}$ is a Hall basis of $L$, then the right hand side has as basis the disjoint union

$$
\bigcup_{n / 2<k<2 n / 3}\left\{u \otimes w \mid u \in \mathcal{H} \cap L_{k}, w \in \mathcal{H} \cap L_{n-k}\right\}
$$

and this basis is mapped by $u \otimes w \mapsto[u, w]$ one-to-one into $\mathcal{H}$, with the image spanning the left hand side.) These sums have at least $\frac{n}{6}-1$ summands. If $v$ is a partition of $n$ other than $(n),(n-1,1),\left(2,1^{n-2}\right),\left(1^{n}\right)$, then [v] does occur in each of these summands. It follows that the multiplicity of such $a[v]$ in $L_{n}$ is at least $\frac{n}{6}-1$ (provided of course that $v$ has no more than $\operatorname{dim} V$ parts). In particular, if a multiplicity in $L_{n}$ is larger than 1 , then it is at least $\frac{n}{6}-1$.

Added in proof (March 8, 2006). Since this paper was submitted, Marianne Johnson at the University of Manchester has been able to deduce Klyachko's Theorem directly from the Kraśkiewicz-Weyman Theorem ('Standard tableaux and Klyachko's Theorem on Lie representations', J. Combin. Theory Ser. A, to appear).

Acknowledgments. We are indebted to the referee for helpful suggestions and for pointing out that Lemma 1 is equivalent to a special case of Theorem 3.3 in Sundaram [11]. This work was carried out while the second author was visiting the Australian National University. Financial support from EPSRC (Overseas Travel Grant GR/S70586/01) is gratefully acknowledged.

\section{References}

1. Angeline J. Brandt, “The free Lie ring and Lie representations of the full linear group," Trans. Amer. Math. Soc. 56 (1944), 528-536.

2. Marshall Hall, Jr., "A basis for free Lie rings and higher commutators in free groups," Proc. Amer. Math. Soc. 1 (1950), 575-581.

3. Torsten Hannebauer and Ralph Stöhr, "Homology of groups with coefficients in free metabelian Lie powers and exterior powers of relation modules and applications to group theory," in Proc. Second Internat. Group 
Theory Conf. (Bressanone/Brixen, June 11-17, 1989), Rend. Circ. Mat. Palermo (2) Suppl. 23 (1990), 77-113.

4. A.A. Klyachko, "Lie elements in the tensor algebra," Sibirsk Mat. Ž. 15(1974), 1296-1304, 1430 (Russian). English translation: Siberian J. Math. 15 (1974), 914-921 (1975).

5. Witold Kraśkiewicz and Jerzy Weyman, "Algebra of coinvariants and the action of a Coxeter element," Bayreuth. Math. Schr. No. 63 (2001), 265-284 (Preprint, 1987).

6. Frank Levin, "Generating groups for nilpotent varieties," J. Austral. Math. Soc. 11 (1970), 108-114.

7. I.G. Macdonald, Symmetric functions and Hall polynomials, Oxford University Press, Oxford, 1979.

8. Cristophe Reutenauer, Free Lie algebras, Oxford University Press, Oxford, 1993 (London Math. Soc. Monographs, New Ser., Vol. 7).

9. Manfred Schocker, Über die höheren Lie-Darstellungen der symmetrischen Gruppen, Dissertation, Kiel, 2000; Bayreuth. Math. Schr. No. 63 (2001), 103-263.

10. Manfred Schocker, "Embeddings of higher Lie modules," J. Pure Appl. Algebra 185 (2003), $279-288$.

11. Sheila Sundaram, "Decompositions of $S_{n}$-submodules in the free Lie algebra," J. Algebra 154 (1993), 507-558.

12. R.M. Thrall, "On symmetrized Kronecker powers and the structure of the free Lie ring," Amer. J. Math. 64 (1942), 371-388.

13. M.R. Vaughan-Lee, "Generating groups of nilpotent varieties," Bull. Austral. Math. Soc. 3 (1970), 145154.

14. F. Wever, "Über Invarianten von Lieschen Ringen," Math. Ann. 120 (1949), 563-580.

15. V.M. Zhuravlev, "The free Lie algebra as a module over the general linear group," Mat. Sb. 187(2) (1996), 59-80 (Russian). English translation: Sb. Math. 187(2) (1996), 215-236. 